ISSN: $1782-2041$

DOI: $10.25518 / 1782-2041.1166$

\title{
Motivation et normativité : Réflexions sur le concept de liberté à partir de Merleau-Ponty
}

Par JAKUB ČAPEK

Université Charles de Prague

\section{Introduction : les différentes facettes de la motivation}

Le concept de «motif» constitue un aspect indispensable de l'analyse philosophique de l'agir humain. Alléguer un motif, c'est donner la réponse à la question du " pourquoi ? » de l'action. Or nous avons plusieurs façons de donner une telle réponse. Certains suggèrent que la motivation est indissociablement liée à la normativité. Pour commencer, nous allons donc établir une distinction entre deux concepts de motif.

\subsection{Deux concepts de motif}

Lorsqu'on parle du motif d'une action, il faut distinguer plusieurs choses, comme le suggère Elisabeth Anscombe dans son livre L'Intention ${ }^{1}$. On peut évoquer un état de choses antérieur à l'action (un motif rétrospectif), ou bien une tendance générale (un motif général ou interprétatif), ou bien encore un état de choses désiré (un motif prospectif, la fin de l'action). Si l'on me demande pourquoi j'assiste à une conférence, je peux donner trois réponses différentes. Ou bien, je peux dire «parce qu'elle a été annoncé », ou bien «par curiosité », ou encore « pour enrichir mes connaissances dans le domaine qui fait son objet ». Les trois réponses peuvent être vraies à la fois, mais chacune

${ }^{1}$ E. Anscombe, Intention, Cambridge (MA) London, Harvard University Press, 2000, $\S \S 13$ et 16 . 
se réfère à une autre structure de motivation, à une autre justification de l'action par des raisons d'agir différentes.

Dans les textes qui développent une phénoménologie de l'action, et finalement aussi dans le livre d'Anscombe, le troisième type de réponse tout juste citée n'est pas considéré comme un «motif». On parlera plutôt ici de l'intention, du dessein ou du projet. Sous un motif, Ricœur, Sartre ou MerleauPonty entendent le plus souvent le motif en tant qu'antécédent d'une action. Or, si je dis que j'ai agi «par curiosité » ("par amitié », " par amour de vérité », pour donner d'autres exemples d'Anscombe), je ne mentionne pas un état de choses antérieur, mais j'invite les autres à " voir mon action sous cette lumière », comme l'exprime Anscombe ; je les invite donc à comprendre mon action comme un effet de ma curiosité, un trait de mon caractère, c'est-à-dire comme un cas particulier d'une caractéristique plus générale. De façon similaire, Anthony Kenny décrit ce concept de motif en recourant au terme de « schéma» (pattern, scheme) ${ }^{1}$.

La phénoménologie de la motivation - que l'on peut trouver chez Ricœur depuis Le Volontaire et l'involontaire jusqu'au Soi-même comme un autre, et chez Merleau-Ponty dans la Phénoménologie de la perception - caractérise avant tout le motif comme un antécédent de l'agir ; mais dans certains contextes, elle le définit aussi comme un trait général de l'agir (un trait de caractère, une tendance personnelle, sociale ou humaine). Dans ce qui suit, je vais montrer qu'il y a, dans la Phénoménologie de la perception, deux conceptions différentes de la motivation. Pour commencer, je vais distinguer les deux concepts du motif et montrer qu'ils impliquent des observations différentes concernant la liberté.

\subsection{Le motif comme antécédent de l'action}

Si l'on s'intéresse au motif comme à un état de choses qui précède l'action, comme ce qui nous incite à agir dans une situation donnée, on doit distinguer le motif - par exemple, la faim ou la fatigue — d'une cause. Même si j'ai faim, même si je suis fatigué, je ne suis pas forcé d'agir dans le sens indiqué ; l'action n'est pas entraînée par la motivation. Dans Le Volontaire et l'involontaire, Paul Ricœur l'énonce de façon claire et nette : le motif n'est pas une

${ }^{1}$ A. Kenny, Action, Emotion and Will, London, Routledge and Kegan Paul, 1963, p. $90-98$. 
cause. Il ajoute : «Le propre d'une cause est de pouvoir être connue et comprise avant ses effets $\gg{ }^{1}$, ce qui ne vaut pas pour le motif, bien au contraire. Un motif n'a pas « de sens complet en dehors de la décision qui l'invoque. Il ne m'est pas donné de comprendre d'abord et en eux-mêmes les motifs, et d'en dériver secondement l'intelligence de la décision $»^{2}$. On retrouve le même argument dans différents textes de la philosophie analytique, par exemple sous la forme : la cause et l'effet sont logiquement indépendants, tandis qu'il est impossible d'assurer une pareille indépendance logique pour le rapport entre le motif et l'action ${ }^{3}$. Autrement dit, le rapport causal est extensionnel, « il existe entre les termes sans qu'on doive tenir compte de la manière dont les termes nous sont présentés ${ }^{4}$; en d'autres termes, il s'agit d'un rapport entre les événements eux-mêmes. Cependant lorsqu'il s'agit d'un rapport de motivation, il est nécessaire de se référer à la manière dont le rapport est décrit. Nous ne le comprenons qu'en « le décrivant de la manière dont il existe pour l'agent $»^{5}$. Bref, cet argument, présenté sous des formes diverses, signale que le motif en tant que motif n'existe pas en dehors du rapport à l'action naissante. C'est pourquoi il est exclu de concevoir ce rapport comme une succession réelle de deux événements dont l'un serait la cause de l'autre. Ricœur trouve l'essence de la motivation dans la formule « le motif incline sans nécessiter $»^{6}$, et il affirme qu'il y a un rapport réciproque entre le motif et l'action.

\footnotetext{
${ }^{1}$ P. Ricœur, Philosophie de la Volonté. Le Volontaire et l'involontaire, Paris, Aubier, 1988, p. 65.

${ }^{2}$ P. Ricœur, Philosophie de la Volonté, op. cit., p. 65 ; cf. aussi P. Ricœur, « L'Unité du volontaire et de l'involontaire comme idée-limite », Bulletin de la Société Française de Philosophie nº5/1 (1951), p. 7 : «[I]1 n’y a pas de motif en dehors de ce rapport réciproque avec une décision naissante».

${ }^{3}$ Cf. par exemple G.H. von Wright, Explanation and Understanding of Action dans S. Körner [dir.], Practical Reason, Oxford, Blackwell, 1983, p. 61 sq. ; pour d'autres références, $c f$. D. Davidson, Essays on Actions and Events, Oxford, Clarendon Press, 1980, p. 13, n. 6.

${ }^{4}$ Cf. M. A. Wrathall, Motives, Reasons, and Causes, dans R. Carman et al. [dir.], The Cambridge Companion to Merleau-Ponty, Cambridge, Cambridge University Press, 2005, p. 119 sq : "Causal relations [...] are extensional in the sens that the relationship holds between the relata regardless of the mode by which the relata are presented to us ».

${ }^{5}$ M. A. Wrathall, Motives, Reasons, and Causes, op. cit., p. 120 : «We only capture the motivational relationship if we describe the relationship as it exists for the agent ». ${ }^{6}$ P. Ricœur, Le Volontaire et l'involontaire, op. cit., p. 69. Cette formule est sans doute reprise de G.W. Leibniz, Discours de métaphysique dans H.H. Holz [dir.], Philosophische Schriften, Darmstadt, 1985, vol. 1, p. 84 (XIII) : « [L]eur choix [= celui des créatures] a toujours ses raisons, mais elles inclinent sans nécessiter ».
} 
Merleau-Ponty recourt à des observations analogues lorsqu'il déclare par exemple que « les motifs n'annulent pas la liberté » ${ }^{1}$. Lui aussi développe en outre des analyses de la motivation dans lesquelles « motif » signifie « état de choses antécédent à l'action ». Il écrit par exemple :

Qu'entend-on par un motif et que veut-on dire quand on dit, par exemple, qu'un voyage est motivé ? On entend par là qu'il a son origine dans certains faits donnés, non que ces faits à eux seuls aient la puissance physique de le produire, mais en tant qu'ils offrent des raisons de l'entreprendre. Le motif est un antécédent qui n'agit que par son sens [...]. Motif et décision sont deux éléments d'une situation : le premier est la situation comme fait, le second la situation assumée. Ainsi un deuil motive mon voyage. [...] En décidant de faire ce voyage, je valide ce motif qui se propose et j'assume cette situation ${ }^{2}$.

Ainsi, chez Merleau-Ponty et Ricœur, le rapport de motivation n'est pas causal parce que la motivation ne peut faire l'économie d'une certaine compréhension : le motif est un antécédent qui « n'agit que par son sens ». Merleau-Ponty finit par désigner lui aussi ce rapport entre le motif et la décision comme un rapport réciproque et circulaire : « La relation du motivant et du motivé, écritil par exemple, est donc réciproque $»^{3}$. Il ajoute à cette déclaration une observation importante : cette détermination mutuelle est un devenir qui laisse surgir une organisation. Le rapport de motivation est décrit comme un passage de l'indéterminé au déterminé, comme la cristallisation d'une organisation univoque sur le fond d'une situation ambiguë. C'est ce que souligne de manière remarquable le tout premier passage sur la motivation qu'on trouve dans la Phénoménologie de la perception : l'objet, dit Merleau-Ponty, " ne suscite 1'"événement connaissant" qui le transformera, que par le sens encore ambigu qu'il lui offre à déterminer, si bien qu'il en est le "motif" et non pas la cause ${ }^{4}$.

Il en résulte certaines conséquences pour le concept de liberté. Si l'on prend au sérieux l'idée que les actes libres ont toujours un motif, alors on doit d'abord refuser l'idée que la liberté équivaut à l'indétermination — c'est précisément ce que fait Ricœur dans Le Volontaire et l'involontaire, lorsqu'il décrit l'hésitation et le choix ${ }^{5}$. De façon non moins univoque, on doit refuser l'idée d'une liberté absolue, comme le fait Merleau-Ponty dans sa critique de Sartre. Une liberté motivée n'est ni indéterminée, ni absolue. C'est une liberté

\footnotetext{
${ }^{1}$ M. Merleau-Ponty, Phénoménologie de la perception, Paris, Gallimard, 1999, p. 510.

${ }^{2}$ M. Merleau-Ponty, Phénoménologie de la perception, op. cit., p. 299.

${ }^{3}$ Ibid., p. 299 sq.

${ }^{4}$ Ibid., p. 39.

${ }^{5}$ P. Ricœur, Le Volontaire et l'involontaire, op. cit., p. 66 sq, 155-186.
} 
en situation, comme le remarque Merleau-Ponty dans sa critique de Sartre : «L'idée de situation exclut la liberté absolue à l'origine de nos engagements ${ }^{1}$. Mais — et c'est ce que je vais proposer dans la suite de mon exposé - l'argument le plus important de sa critique de Sartre ne s'appuie pas sur l'analyse du motif comme antécédent à l'action, mais sur l'analyse du motif en général. Ceci nous amène à considérer le deuxième sens du concept de «motif ».

\subsection{Le motif en général}

Lorsque nous expliquons notre action à autrui, nous pouvons dire que nous l'avons entreprise «par curiosité », «par amour de la nature », «par habitude », etc. Dans ces cas-là, nous évoquons une tendance ou un trait personnel qui est général par rapport à des cas concrets. Ce trait général comporte souvent un aspect évaluatif : c'est parce que j'aime la nature en général (motif général) que j'ai accepté l'invitation de faire une randonnée (le motif comme antécédent de l'action). Ce n'est qu'à la lumière d'un tel motif général que l'on peut décider si cette invitation est à accepter ou pas. Le motif particulier (en tant qu'il précède à l'action) ne permet pas à lui seul de déterminer ce que j'ai à faire.

Lorsque Merleau-Ponty refuse la doctrine sartrienne de la liberté, il s'appuie sur une certaine analyse de la généralité de la motivation. Sartre affirme que la liberté est, « totale et infinie $»^{2}$. Il est vrai qu'une décision de couper court une promenade dans les montagnes peut être motivée par une fatigue de plus en plus insupportable. Mais, selon la description sartrienne bien connue, nous décidons que ce motif (la fatigue) a la force de changer notre projet. Nous conférons à la fatigue la force de nous motiver. Dans une telle analyse, selon Merleau-Ponty : "L'idée de l'action disparaît». Pour lui, "l'action libre, pour être décelable, devrait se détacher sur un fond de vie qui ne le fût pas ou qui le fût moins $»^{3}$. La motivation devrait donc pouvoir indiquer comment agir et proposer certaines directions plutôt que d'autres :

\footnotetext{
${ }^{1}$ M. Merleau-Ponty, Phénoménologie de la perception, op. cit., p. 518.

2 J.-P. Sartre, L'Être et le Néant, Paris, Gallimard, 1999, p. 576.

${ }^{3}$ M. Merleau-Ponty, Phénoménologie de la perception, op. cit., p. 499.
} 
S'il n'y a pas des cycles de conduite, de situations ouvertes qui appellent un certain achèvement et qui puissent servir de fond, soit à une décision qui les confirme, soit à une décision qui les transforme, la liberté n'a jamais lieu ${ }^{1}$.

Merleau-Ponty exprime en une phrase cette idée que la liberté doit pouvoir « se détacher sur un fond de vie » qui doit être décelable : « La liberté, dit-il, doit avoir du champ », un lieu où « des possibles privilégiés $»^{2}$ se dessinent.

Mais comment les situations elles-mêmes peuvent-elles « appeler » un certain achèvement? Comment des possibilités peuvent-elles être déjà triées, donc séparées en "privilégiées » et non privilégiées ? Pour Merleau-Ponty, c'est concevable à condition qu'on ne raccorde pas ces possibilités au projet individuel, mais à la " valorisation spontanée ", à une évaluation spontanée de mon entourage de sorte que « je porte autour de moi des intentions qui ne sont pas décisoires et qui affectent mon entourage de caractères que je ne choisis pas $»^{3}$. Merleau-Ponty poursuit l'analyse de cette valorisation spontanée, ancrée en nous sans être pour autant notre propre prise de position : elle est fondée, soit dans notre corps, soit dans notre histoire personnelle ou collective. Autant que mon corps, mon histoire précède mes prises de position et me dispose à quelque chose.

C'est à travers le thème de la valorisation spontanée que Merleau-Ponty entame une tout autre analyse de la motivation et de la liberté. La motivation générale est saisie comme une capacité d'évaluer notre entourage qui ne se réfère pas à notre décision explicite. Même si cette évaluation n'est pas quelque chose que nous faisons, mais quelque chose qui se fait en nous, ou à travers nous, elle nous dispose à discriminer un comportement "propre », « adéquat» ou « bon », d'un comportement qui ne l'est pas. Bref : la « valorisation spontanée » comporte une dimension normative. Elle montre que notre perception et notre activité se déroule dans un contexte normatif, qu'il y a une normativité latente qui fait indispensablement partie de la perception et de l'action.

\section{La normativité}

Lorsqu'on doit regarder un spectacle à partir d'une place située au fond de la salle, on sait ce qu'il faut faire pour voir mieux : se rehausser ou essayer de changer de place. De manière générale, tout le monde comprend ce que signifie

${ }^{1}$ Ibid., p. 500.

${ }^{2}$ Ibid.

${ }^{3}$ Ibid., p. 501 sq. 
« voir mieux » ou « voir bien » (« proprement»). Selon l'analyse développée par Husserl, mais aussi Merleau-Ponty, le « voir mieux » renvoie à une norme de et pour la vision. C'est à la lumière de cette norme que la vision peut être jugée bonne, mauvaise, voire insuffisante ${ }^{1}$. Dans ce qui suit, je vais développer l'idée selon laquelle la motivation comporte des dimensions normatives, et dans le domaine de la perception, et dans celui de l'action.

Pour saisir cette normativité perceptive (et pratique), je vais me tourner d'abord vers les analyses de l'optimum perceptif que l'on trouve chez Husserl et Merleau-Ponty. Ensuite, je vais traiter le thème de la concordance ou de la continuité comme un aspect normatif de l'expérience. Je m'appuierai alors sur une distinction, que l'on trouve chez Anthony Steinbock, entre deux définitions du normatif : le normatif comme optimal et le normatif comme concordant $^{2}$. Au cours de la dernière décennie, plusieurs auteurs - comme par exemple Steven Crowell, Sean D. Kelly, A. Steinbock, J. Taipale ou M. Doyon - ont attiré l'attention sur la normativité de la perception ${ }^{3}$. Il faut ajouter que lorsqu'on décrit la dimension normative de la perception, on se réfère le plus souvent à un concept large de normativité : est normatif tout ce qui peut servir de standard ou de mesure pour un succès ou un échec ${ }^{4}$. Après avoir considéré les deux formes de la normativité - l'optimal et le concordant - je vais me tourner vers le thème de la "valorisation spontanée » chez Merleau-Ponty.

1 M. Doyon, Perception and Normative Self-Consciousness dans M. Doyon et T. Breyer [dir.], Normativity in Perception, New-York, Palgrave Macmillan, 2015, p. 44 : " Our capacity of self-correction is [...] indeed a good indicator that we find ourselves in a normative space ».

2 A. J. Steinbock, Home and Beyond. Generative Phenomenology after Husserl, Evanston, Northwestern University Press, 1995, p. 129-147.

${ }^{3}$ Pour Crowell, Steinbock et Doyon, voir les notes ici, infra et supra. Pour les autres auteurs, voir par exemple S. D. Kelly, The Normative Nature of Perceptual Experience dans B. Nanay [dir.], Perceiving the World, Oxford, Oxford University Press, 2010, p. 146-159 ; J. Taipale, Phenomenology and Embodiment. Husserl and the Constitution of Subjectivity, Evanston, Northwestern University Press, 2014, p. 121 sq.

${ }^{4} \mathrm{~S}$. Crowell, Normativity and Phenomenology in Husserl and Heidegger, Cambridge, Cambridge University Press, 2013, p. 2 : « There is a wider sense according to which a norm is anything that serves as a standard of success or failure of any kind, and it is in this sense that I understand the term here. Thus a legislated statute is a norm, as are rules of game like chess or baseball ; but "unspoken" rules, satisfaction conditions, culture mores, manners, what is "normally" done - in short, whatever it is that measures our speech and behavoir — are also norms ». Cf. aussi M. Kusch, Knowledge by Agreement, Oxford, Oxford University Press, 2002, ch. 14 : Normativity and Community, p. 175 : «A phenomenon is normative if it essentially involves the distinctions "correct versus incorrect" and "seems right versus is right"».

\section{4}

Bull. anal. phén. XVI 2 (2020)

https://popups.uliege.be/1782-2041/ @ 2020 ULiège BAP 
Pour anticiper la conclusion de ce texte, on peut dire que cette approche suggère une autre définition de la liberté. Une fois qu'on considère la normativité inhérente à la motivation, la question de liberté se pose en effet d'une nouvelle façon : la liberté n'est plus alors définie par rapport à une cause (les motifs « inclinent sans nécessiter »), mais par rapport à une régularité qui s'est établie dans notre comportement et qui exerce une force normative sur notre perception et notre action.

\subsection{Le normatif comme optimal}

Revenons à la question de ce que signifie « mieux voir» ou «bien voir ». Toute perception peut être rapportée à une « perception privilégiée », dit Merleau-Ponty qui poursuit en affirmant que «nous tendons alors vers le maximum de visibilité ${ }^{1}$. Or, qu'est-ce qui définit la perception privilégiée ? Les textes en question, donc avant tout les Idées II et la Phénoménologie de la perception, explorent trois réponses possibles. La perception privilégiée est definie :

$1^{\circ}$ par la chose perçue qui, par exemple, demande à être vue d'une certaine distance. Il s'ensuit que la chose perçue est non seulement le «motif » de certains comportements perceptifs, mais aussi leur mesure. Merleau-Ponty donne l'exemple de la perception du corps vivant : « Le corps vivant lui-même apparaît quand sa microstructure n'est ni trop, ni trop peu visible, et ce moment détermine aussi sa forme et sa grandeur réelle $»^{2}$. Husserl, quant à lui, ne dit pas autre chose. Selon lui, la perception privilégiée est une donation de la chose par laquelle on arrive à ce qui est « relativement le meilleur de la chose $n^{3}$.

$2^{\circ}$ par le corps de celui qui perçoit; en effet, ce sont les sens de cet organisme percevant qui déterminent les qualités sensibles de la chose perçue.

\footnotetext{
${ }^{1}$ M. Merleau-Ponty, Phénoménologie de la perception, op. cit., p. 348.

2 Ibid., p. 349.

${ }^{3}$ E. Husserl, Idées directrices pour une phénoménologie et une philosophie phénoménologique pures. Tome 2 : Recherches phénoménologiques pour la constitution, trad. fr. É. Escoubas, Paris, PUF, 1982, p. 60 : «Ainsi dans la série des apparitions possibles, une donnée de chose a donc ce privilège, qu'avec elle se donne ce qui est relativement le meilleur de la chose en général et c'est elle qui reçoit le caractère de la donnée qui est tout aussi particulièrement en rapport avec l'intention ; c'est sur elle que se dirige 1'“intérêt" de façon prépondérante, c'est en elle que la tendance de l'expérience trouve son terme, se remplit et les autres modes de donnée entretiennent un rapport intentionnel avec la donnée "optimale"».
} 
Par exemple, il n'y a de perception privilégiée de la couleur d'une chose que pour un être capable de percevoir des couleurs. Le corps détermine, selon Husserl et Merleau-Ponty, non seulement la manière dont les choses se montrent à nous, mais aussi les conditions sous lesquelles elles se montrent bien ou proprement. Husserl écrit à ce propos : « La complexion [die Beschaffenheit] des choses matérielles en tant qu'aistheta, telle qu'elles se présentent à moi dans l'intuition, dépend de la complexion du sujet de l'expérience [abhängig sind von meiner, des erfahrenden Subjekts, Beschaffenheit], c'est-à-dire de mon corps et de ma "sensibilité normale"»".

$3^{\circ}$ par le processus perceptif lui-même, et, plus exactement, par certains traits de ce processus : sa cohérence et sa continuité. Au cours du déroulement perceptif, nous pouvons éprouver un enchaînement continu aussi bien qu'une rupture. Chez Husserl et Merleau-Ponty, c'est le caractère concordant du processus perceptif qui détermine ce que signifie « bien voir », c'est-à-dire l'optimum perceptif.

Ces trois réponses doivent être analysées ensemble car il s'agit des trois aspects d'une même corrélation. La première réponse - l'optimum est défini par la chose elle-même - n'explique pas l'intérêt qui nous guide au cours de la perception et qui co-détermine ce qui vaut comme "la chose même ». La deuxième réponse - l'optimum perceptif est défini par le corps percevant n'explique pas les conditions sous lesquelles un corps percevant peut être pris pour « normal ». Sans entrer en détail, je propose à titre d'hypothèse que c'est la troisième réponse qui assure la pierre de touche de la phénoménologie de la normativité perceptive chez Husserl et Merleau-Ponty. Une même chose, la porte de la salle par exemple, peut se trouver dans l'horizon de mon champs visuel, ou bien en son centre ; elle peut être donnée à ma vue, mais aussi à mon toucher. Entre ces différentes manières d'éprouver la même chose, il peut y avoir soit un enchaînement continu, soit une rupture ou une discordance. Je crois que c'est précisément ici qu'Husserl et Merleau-Ponty localisent la source de la normativité perceptive : la perception est une invitation à voir le monde en continu et en cohérence ses différents aspects. Husserl est très clair sur ce point : "Le "faux" réside dans la contradiction avec le système normal de l'expérience $»^{2}$.

\footnotetext{
${ }^{1}$ E. Husserl, Ideen II dans Husserliana IV, Haag, Nijhoff, 1952, p. 56 : « Es stellt sich heraus, daß die Beschaffenheit der materiellen Dinge als Aistheta, so wie sie anschaulich vor mir stehen, abhängig sind von meiner, des erfahrenden Subjekts, Beschaffenheit, bezogen auf meinen Leib und meine "normale Sinnlichkeit"».

${ }^{2}$ Ibid., p. 61.
} 
Conclusion provisoire : c'est le déroulement perceptif, et, plus précisément, son caractère cohérent ou concordant, qui rend possible la désignation, au cours d'une expérience concrète, d'une certaine perception comme privilégiée.

\subsection{Le normatif comme concordance}

Il faut maintenant envisager une définition du normatif d'après le prédicat de la concordance en nous demandant d'où vient le prestige de la concordance. Qu'est-ce qui justifie cette valeur normative de la concordance ? Le constat initial d'Husserl et Merleau-Ponty se laisse résumer par l'idée que " toute expérience a un horizon ». Pour les deux philosophes, il s'agit là d'une affirmation descriptive, et non pas normative.

Dans Expérience et Jugement, un volume édité par Ludwig Landgrebe, Husserl définit « l'expérience au sens premier et authentique » comme la « donation d'objets individuels dans leur ipséité », c'est-à-dire " en personne » ${ }^{1}$. Or, la donation «en personne » n'épuise pas le concept d'expérience. Pour éprouver quelque chose, un objet, une personne ou un événement, tel qu'un spectacle théâtral, il faut savoir comment l'approcher. Chaque expérience est rendue possible par un pré-savoir (Vorwissen) que Husserl explique par le terme « horizon »: «Toute expérience, écrit-il, a son horizon propre $»^{2}$. Les expériences possibles d'un objet perçu se situent «à l'horizon » de l'expérience présente. Il s'agit des expériences que nous pouvons ou savons faire. Il y a selon Husserl une corrélation entre, d'une part, le déroulement possible de l'expérience, et, d'autre part, les possibilités ou les capacités de la personne qui fait cette expérience ${ }^{3}$. Cet ensemble de capacités ou de pouvoirs de celui

\footnotetext{
${ }^{1}$ E. Husserl, Erfahrung und Urteil, Prague, Academia, 1939, § 6, p. 21; Expérience et Jugement, trad. fr. D. Souche-Dagues, Paris, PUF, 2011, p. 30. Sur la définition husserlienn du concept d'expérience, on pourra également lire Erfahrung und Urteil, op. cit., § 7, p. 23 (« Erfahrung als Selbstgebung individueller Gegenstände »); Formale und transzendentale Logik, Halle, Niemeyer, 1929, § 84 ; Ideen zu einer reinen Phäenomenologie und phänomenologischen Philosophie I, Haag, Nijhoff, 1976, § 1 ( « ein Reales originär gegeben haben ») et $\$ 3$ ( « in seiner "leibhaftigen Selbtsheit"»). ${ }^{2}$ E. Husserl, Erfahrung und Urteil, op. cit., § 8, p. 27 : « Jede Erfahrung hat ihren Erfahrungshorizont ».

${ }^{3}$ Ibid., p. 27. Lorsqu'Husserl explique son idée que « toute expérience a son horizon propre », il ajoute que l'expérience " renvoie à la possibilité » («verweist auf die Möglichkeit ») qui est corrélative d'une capacité ou d'un pouvoir de moi-même ( « und vom Ich her eine Ver-möglichkeit »).
} 
qui éprouve quelque chose peut lui aussi porter le nom d' « expérience»; voici pourquoi il faut distinguer deux conceptions différentes de l'expérience : une conception cognitive, suivant laquelle l'expérience désigne tout d'abord la connaissance de quelque chose, et une conception dispositionnelle pour laquelle l'expérience renvoie plutôt aux dispositions de celui qui fait l'expérience au sens cognitif. ${ }^{1}$ Suivant cette deuxième acception (l'expérience comme disposition), on peut même caractériser une personne comme « celui ou celle qui a de l'expérience ». À lire d'ailleurs Expérience et Jugement, cette seconde conception de la notion d' " expérience » est plus proche de l'usage quotidien et familier du terme :

[C]e sens courant [du mot expérience] ne se rapporte d'aucune manière purement et simplement à la conduite cognitive, mais, pris dans sa plus grande généralité, à une habileté qui confère à celui qui en est doué, à celui qui «a de l'expérience », la sûreté de la décision et de la pratique dans les situations de la $\mathrm{vie}^{2}$.

La deuxième conception de l'expérience se réfère à la capacité d'agir, de décider, et, de manière générale, d'éprouver quelque chose. La possibilité pour une chose d'être connue est corrélative de ma capacité à la connaître. Nous pouvons «avoir de l'expérience » non seulement dans le domaine de la délibération et de l'action, mais aussi dans celui de la perception. Comme Hubert Dreyfus et Charles Taylor l'expriment dans leurs lectures de Merleau-Ponty, en un certain sens, nous sommes toujours déjà, même sans y penser, des experts de la perception. ${ }^{3}$

Pour revenir au thème de la continuité de l'expérience, nous pouvons dire que c'est le caractère dispositionnel ou habituel de l'expérience qui rend possible l'anticipation du déroulement prochain de notre expérience. Sans lui,

${ }^{1}$ C'est l'expérience au sens dispositionnel qui est au centre de la pensée de HansGeorg Gadamer dans Vérité et Méthode. Pour le sens dispositionnel de l'expérience, cf. aussi L. Tengelyi, The Wild Region in Life-History, Evanston, Northwestern University Press, 2004, p. 7.

${ }^{2}$ E. Husserl, Erfahrung und Urteil, op. cit., § 12, p. 52.

${ }^{3}$ Cf. H. Dreyfus et C. Taylor, Retrieving Realism, Cambridge (MA) London, Harvard University Press, 2015, p. 61-62: «[I]n perception, as in action, we are experts in viewing objects ». Pour les limites de cette lecture de Merleau-Ponty, cf. J. Čapek, Perceptual Faith beyond Practical Involvement : Merleau-Ponty and His Pragmatist Readers dans O. Švec et J. Čapek [dir.], Pragmatic Perspectives in Phenomenology, New York, Routledge, 2017, p. 141-157. 
notre expérience ne serait pas continue. C'est l'expérience au sens dispositionnel - l'expérience comme capacité - qui contribue à créer l'horizon ou le cadre dans lequel nous comprenons les choses et les événements, soit comme « familiers », soit comme « surprenants ». Cet horizon est toujours retravaillé, toujours « en mouvement $»^{1}$. Chaque nouvelle expérience crée de nouvelles anticipations, même « sans notre participation active ou explicite ». ${ }^{2}$

Parce que nous sommes déjà initiés à l'expérience avec ses horizons, le déroulement de notre expérience a le caractère d'une confirmation ou d'une réfutation continue de nos anticipations ${ }^{3}$. C'est donc finalement l'idée de l' ' horizon » comme partie indispensable de toute expérience qui explique le prestige de la continuité et de la concordance de l'expérience ${ }^{4}$. La continuité et la concordance sont avant tout des traits descriptifs. Mais il s'avère que, chez Husserl et Merleau-Ponty, ils reçoivent un statut normatif.

Il faut bien voir qu'Husserl et Merleau-Ponty comprennent différemment cette normativité de la concordance. Chez Husserl, la concordance est saisie à la lumière d'un idéal cognitif : l'objet lui-même. Selon lui, « l'objet qui serait déterminé de part en part, connu de part en part $»^{5}$ est une idée qui guide toute perception. Il s'agit, bien sûr, d'une "idée située dans l'infini », « l'idée d'un soi-même absolu de l'objet et de sa déterminité absolue et complète ». Au regard de cette idée, toute perception n'est qu'une approximation. Très souvent, le donné optimal est déterminé par un contexte pratique, c'està-dire par rapport à un " intérêt » qui est « chaque fois en jeu ». Une maison perçue de façon optimale, par exemple, n'est pas une donnée absolue ou complète, mais une donnée relativement complète :

\footnotetext{
${ }^{1}$ E. Husserl, Erfahrung und Urteil, op. cit., § 25, p. $136 \mathrm{f}$.

${ }^{2}$ Ibid., § 25, p. $137 \mathrm{f}$.

${ }^{3}$ Ibid., p. 27 : « Toute expérience peut être étendue en une chaîne continue d'expériences singulières explicatrices ». Pour un définition analogue, $c f$. M. Merleau-Ponty, Phénoménologie de la perception, op. cit., p. 82 sq.

${ }^{4}$ Cette idée est résumée par M. Doyon, Perception and Normative Self-Consciousness, op. cit., p. 39 : «In Husserl's view, it is in this horizontal structure that perception acquires its normative character, for it is only against the background of these fulfilled or unfullfilled expectations that perceptual content can be said to be accurate or inaccurate, correct or incorrect, better or worse, just fine or optimal, etc. ».

${ }^{5}$ Cette citation et les citation suivantes sont extraites de cette référence : E. Husserl, De la synthèse passive. Logique transcendantale et constitutions originaires, trad. fr. B. Bégout, N. Depraz et M. Richir, Grenoble, Millon, 1998, p. 109-110. Cf. aussi E. Husserl, Ideen I, op. cit., § 143.
} 
[L]a maison elle-même est-elle très rapidement donnée de façon optimale [...], donc complètement expérimentée par celui qui la considère en tant qu'acheteur ou vendeur. Pour le physicien et le chimiste, un tel mode d'expérience semblerait totalement superficiel et encore à des années-lumière du véritable être ${ }^{1}$.

Même si Husserl admet que, en contexte pratique, l'optimum perceptif n'est que relatif, il n'en reste pas moins que, selon lui, notre expérience est guidée par une idée limite qui est celle d'une donation optimale de la chose ellemême. Il s'agit bien d'une idée "située dans l'infini ». C'est cette idée qui nous permet de dire qu'une autre donation, qu'une meilleure présentation de la chose, n'est pas exclue ${ }^{2}$.

Chez Merleau-Ponty, par contre, cet idéal d'une donation complète et absolue ne fait pas partie de la perception. S'il y a une normativité immanente à la perception qui nous permet d'expliquer ce que signifie « voir mieux », ce n'est pas à un idéal quasi epistémologique qu'elle pourrait être assimilée. Il est vrai que nous somme capables d'incorporer des anticipations et de les transporter sur nos expériences ultérieures, mais il ne s'agit pas ici d'un désir épistémique (d'une tendance à aller du vide au plein). La perception est un comportement existentiel ou pragmatique. Toute perception optimale est relative à notre corps en tant qu'être vivant, en tant qu'organisme qui doit toujours rétablir son equilibre corporel. Le sujet perçevant est un sujet pratique. Certains interprètes de Merleau-Ponty suggèrent que c'est finalement les " pratiques » qui expliquent la normativité perceptive ${ }^{3}$. Les conditions du succès et de l'échec d'une perception sont finalement identiques aux conditions du succès et de l'échec d'une activité comme " marcher», " regarder », " courir», etc. « Voir bien » ou « voir mieux » signifie pour Merleau-Ponty avoir une « prise sur un monde », être capable de « s'installer dans son monde » ${ }^{4}$, et agir en lui. Il conçoit la perception non comme une tendance qui va du vide au plein, mais comme une orientation dans le monde et comme une « communication ou une communion $»^{5}$ avec lui. Cette orientation comporte déjà des structures évaluatives, que le dernier chapitre de la Phénoménologie de la perception caractérise en termes de « valorisations spontanées ».

\footnotetext{
${ }^{1}$ E. Husserl, De la synthèse passive, op. cit., p. 112.

${ }^{2}$ Ibid., p. 110.

3 S. Crowell, Normativity and Phenomenology in Husserl and Heidegger, op. cit., p. 143.

${ }^{4}$ M. Merleau-Ponty, Phénoménologie de la perception, op. cit., p. 349-350.

${ }^{5}$ Ibid., p. 370.
} 


\subsection{La valorisation spontanée}

Dans la dernière partie de cet exposé, je vais me pencher sur le thème de motivation dans son rapport à la question de la liberté. Je vais revenir encore sur le dernier chapitre de la Phénoménologie de la perception, intitulé « La liberté », où Merleau-Ponty refuse l'idée sartrienne de la liberté absolue, c'està-dire l'idée suivant laquelle la liberté ferait surgir les obstacles et les motifs de l'action. À la liberté absolue, Merleau-Ponty oppose l'idée d'une liberté « concrète et effective » qui s'élabore dans un «échange » avec le monde, c'est-à-dire en reprenant " quelques propositions du monde », en vivant des situations qui « appellent un certain achèvement $»^{1}$. Pour une liberté « concrète et effective », il y a "des possibles privilégiés ou des réalités qui tendent à persévérer dans l'être $»^{2}$. Or, pour qu'une situation puisse m'inviter à me comporter d'une certaine façon, elle doit être comprise ou évaluée. Pour décrire cette évaluation, Merleau-Ponty a recours au concept de « spontanéité ». Il y a selon lui « une valorisation spontanée » qui n'est pas faite par nous, mais qui se fait plutôt « en nous $»^{3}$. Merleau-Ponty examine cette spontanéité sur trois niveaux : au niveau du corps, au niveau de l'histoire individuelle et au niveau de la société, "en nous ». C'est en tant que qu'être corporel, en tant qu'être doté d'un passé unique, et en tant qu'être social, que nous sommes capables d'évaluer notre entourage d'une manière qui n'est pas liée à notre intention volontaire et individuelle. Cette évaluation se joue en dehors de notre liberté.

La valorisation spontanée fonctionne d'abord au niveau du corps ; c'est elle qui, par exemple, détermine la perception privilégiée ou " appropriée » d'un objet. Citons encore une fois la phrase clef de la Phénoménologie de la perception : «En tant que j'ai des mains, des pieds, un corps, un monde, je porte autour de moi des intentions qui ne sont pas décisoires et qui affectent mon entourage de caractères que je ne choisis pas $»^{4}$. Par exemple, la montagne est grande ou petite, non par rapport à mon intention individuelle, mais « par rapport à un niveau qui n'est pas seulement individuel, mais celui de "tout homme" $\rangle^{5}$. Il ne s'agit pas, néanmoins, de traits objectivement valables de tout homme (la taille moyenne par exemple), mais de données corporelles implicitement (habituellement) assumées par nous au cours de notre perception de

${ }^{1}$ Ibid., p. 501. Cf. aussi la page $520:$ «[E]lle ne serait pas liberté sans les racines qu'elle poussa dans le monde ».

${ }^{2}$ Ibid., p. 500.

${ }^{3}$ Ibid., p. 503.

${ }^{4}$ Ibid., p. 502.

${ }^{5}$ Ibid., p. 505. 
l'entourage. Merleau-Ponty montre que ce qu'il appelle le corps habituel (comme différent du corps actuel) assure la sûreté et la continuité de notre comportement quotidien.

Il généralise cette observation en disant qu' « il y a quelque chose d'analogue dans toutes les valorisations $»^{1}$. Le second cas examiné par lui est celui du passé individuel. Il reprend l'analyse sartrienne de la fatigue en tant qu'elle constituerait un "motif» à l'interruption d'une randonnée. Selon Sartre, ce n'est pas la fatigue qui me fait interrompre mon voyage, c'est moi qui décide que cette fatigue est insupportable. Même le complexe d'infériorité se réfère, chez Sartre, au projet, c'est-à-dire à un choix toujours renouvelé par le sujet concerné ${ }^{2}$. Contre cela, Merleau-Ponty propose d'y voir non pas un choix, mais la ténacité d'une certaine manière de se comporter. Il a même recours au langage spinoziste ou ravaissonien lorsqu'il parle d'une tendance « à persévérer dans l'être $»^{3}$. Merleau-Ponty suggère de lier cette ténacité aux phénomènes d'habitualisation et de sédimentation :

Il nous faut bien reconnaître une sorte de sédimentation de notre vie : une attitude envers le monde, lorsqu'elle a été souvent confirmée, est pour nous privilégiée. [...] Ce passé, s'il n'est pas une fatalité, a du moins un poids spécifique, il n'est pas une somme d'événements là-bas, bien loin de moi, mais l'atmosphère de mon présent ${ }^{4}$.

Autrement dit, une fois sédimentée, une certaine attitude par rapport au monde, à la fatigue, devient typique ou habituelle ; elle contribue à articuler notre situation qui « appelle des modes de résolutions privilégiées $»^{5}$, donc des abandons fréquents de projets plus ambitieux. Il est peu probable que la personne en question change. Son comportement est général - c'est-à-dire le même dans des situations différentes - et assez prévisible. Il se prête même à une description statistique : il faut «trouver à la pensée statistique un fondement phénoménologique. Elle appartient nécessairement à un être qui est fixé, situé et investi dans le monde $»^{6}$.

Finalement, c'est en tant que membres d'une classe ou d'une nation, en vivant dans un milieu social, que nous sommes porteurs d'une valorisation spontanée. Le plus souvent, le fait d'être membre d'un certain corps social

${ }^{1}$ Ibid., p. 503-504.

2 J.-P. Sartre, L'Être et le Néant, op. cit., p. 504.

${ }^{3}$ M. Merleau-Ponty, Phénoménologie de la perception, op. cit., p. 500.

${ }^{4}$ Ibid., p. 504-505.

${ }^{5}$ Ibid., p. 505.

${ }^{6}$ Ibid. 
n'est pas un thème explicite de mes réflexions, et pourtant, la société est présente dans mon comportement et dans mes évaluations. Pour Merleau-Ponty, il ne s'agit toutefois pas d'un déterminisme social :

Ce n'est pas l'économie ou la société considérées comme système de forces impersonnelles qui me qualifient comme prolétaire, c'est la société ou l'économie telles que je les porte en moi, telles que je les vis, — et ce n'est pas davantage une opération intellectuelle sans motif, c'est ma manière d'être au monde dans ce cadre institutionnel. J'ai un certain style de vie ${ }^{1}$.

Merleau-Ponty refuse l'idée d'un individu qui se connaît lui-même avant son rapport à d'autres membres de la société. Chaque individu est déjà imprégné dans son rapport à soi-même par « une atmosphère de "socialité"»", par une « zone d'existence généralisée »; chacun, dans sa présence à soi, est « déjà médiatisée par elle $»^{3}$. C'est cette présence de la société en nous qui fait que - de nouveau - certaines situations nous « sollicitent » plutôt dans un sens que dans un autre ${ }^{4}$.

Résumons : selon le dernier chapitre de la Phénoménologie de la perception, il y a trois types de valorisation spontanée. Cette valorisation est liée au corps, au passé et au social. Notre existence corporelle, notre passé individuel et notre milieu social nous suggèrent — chacun à sa manière - un mode d'évaluation de notre entourage, un mode de perception des situations, ainsi qu'une façon privilégiée de nous comporter ${ }^{5}$. Il s'agit de suggestions générales et spontanées. Leur généralité renvoie au fait que les valorisations spontanées suggèrent de voir des situations diverses à la lumière d'un comportement typique. Le même style se propose spontanément pour s'appliquer à plusieurs cas. La spontanéité désigne le statut spécifique de cette valorisation : elle n'est

${ }^{1}$ Ibid., p. 506. Rappelons une analogie lointaine et pourtant importante : la couleur de l'éclairage n'est pas perçue pour elle-même comme une couleur particulière, mais c'est à la lumière de l'éclairage que les objets ont leurs couleurs. L'éclairage, comme dit Merleau-Ponty, est «ce que nous assumons, ce que nous prenons pour norme » (ibid., p. 359).

${ }^{2}$ Ibid., p. 511.

${ }^{3}$ Ibid., p. 513. Cf. aussi la page 415 : « Le social est déjà là quand nous le connaissons ou le jugeons $»$.

${ }^{4}$ Ibid., p. 417 : « [L]a nation et la classe ne sont ni des fatalités qui assujettissent l'individu du dehors ni d'ailleurs des valeurs qu'il pose du dedans. Elles sont des modes de coexistence qui le sollicitent. En période calme, la nation et la classe sont là comme des stimuli auxquels je n'adresse que des réponses distraites ou confuses, elles sont latentes ».

${ }^{5}$ Ainsi, ils remplissent les conditions du « normatif » selon Crowell. 
ni le résultat causal de ma condition corporelle, historique ou sociale, ni l'expression de ma prise de position consciente. La spontanéité de cette valorisation désigne une double indépendance : vis-à-vis d'un déterminisme, et par rapport au vouloir de l'individu. Évidemment, il s'agit ici d'un usage du concept de spontanéité que l'on trouve plutôt chez Ravaisson que chez Kant ${ }^{1}$.

\section{Conclusion : la liberté comme envers de la normativité}

En lisant l'avant-dernière page de la Phénoménologie de la perception, on s'aperçoit qu'elle définit la liberté précisément par rapport à ce « sens spontané ». La liberté est ici définie non pas comme un choix libre, mais comme une « prise de conscience »:

Ma liberté peut détourner ma vie de son sens spontané, mais par une série de glissements, en l'épousant d'abord, et non par aucune création absolue. [...] J'ai reçu avec l'existence une manière d'exister, un style. Toutes mes actions et mes pensées sont en rapport avec cette structure [...]. Et cependant, je suis libre, non pas en dépit ou en deçà de ces motivations, mais par leurs moyens².

Cette dernière citation nous permet de formuler deux remarques en guise de conclusion. $1^{\circ}$ La première d'entre elles porte sur le lien existant entre les concepts de motif et de liberté. Si l'on pense la liberté dans le cadre d'une réflexion sur les motifs comme antécédents de l'agir, on cherche à distinguer le motif de la cause et on aboutit à cette affirmation que « le motif incline sans nécessiter ». Si l'on pense la liberté dans le cadre d'une réflexion sur le motif comme structure normative et répétitive (générale), la liberté est alors définie par rapport à cette régularité, c'est-à-dire par rapport à ce que Merleau-Ponty appelle parfois le « sens spontané » da ma vie. Le terme « motivation » ne désigne pas ici une donnée ou un événement, mais une manière récurrente de répondre à un certain type de stimuli. La liberté ne consiste pas dans le choix d'agir de telle ou telle manière par rapport à un motif concret (précédant l'action), mais dans le "détournement» du sens spontané d'un certain style de vie, ou bien dans l'adhésion à ce style avec une perspective nouvelle. Cette liberté ne s'affirme pas dans un fiat, par une décision; elle se cherche dans « une série de glissements ». La liberté n'est pas quelque chose qui s'établirait

${ }^{1}$ C'est plutôt la spontanéité d'une habitude que la spontanéité d'une action libre. Comme l'écrit F. Ravaisson dans De l'habitude, Paris, PUF, 1999, ch. 1, remarque 3 : «L'habitude se révèle comme la spontanéité dans la régularité des périodes ».

${ }^{2}$ M. Merleau-Ponty, Phénoménologie de la perception, op. cit., p. 519. 
dans un instant donné ; c'est ce qui s'élabore au cours d'une prise de conscience de mes motivations. Ce n'est pas la liberté d'agir, mais la liberté de se dégager ("par une série de glissements»), de détourner la vie « de son sens spontané » ou bien de «l'assumer » d'une manière qui n'est plus seulement spontanée ${ }^{1}$. Évidemment, ces quelques suggestions ne peuvent aucunement prétendre à la définition du concept de liberté. Elle ne font qu'indiquer la direction dans laquelle il faudrait la chercher.

$2^{\circ}$ La deuxième remarque de cette conclusion concerne quant à elle l'argument en faveur de cette liberté. Merleau-Ponty ne fournit pas d'observations concrètes concernant la manière dont on peut acquérir une telle liberté. Ce qu'il nous offre néanmoins, c'est un argument général en faveur de la possibilité d'un tel détournement. Même si la valorisation spontanée n'est pas créée par nous, elle exprime l'expérience en un sens dispositionnel, c'est un savoir faire ou un savoir regarder qui s'est fait, une disposition. Même si nous avons la tendance, la « persévérance » d'éprouver les choses d'une manière, il n'est pas exclu que nous les voyions un jour autrement. Une fois liée à la disposition, la normativité n'est plus quelque chose de purement extérieur à nous. Elle est portée par nous. C'est ce que l'on peut tirer de la lecture des formes différentes de la normativité (optimale et concordante) : il n'y a de normes que pour un être capable de les assumer, de les porter, et, dans certains cas, de s'en détourner. S'il est vrai que la normativité est liée au caractère dispositionnel de notre expérience, c'est-à-dire à notre capacité d'introduire des concordances et des continuités dans ce que nous éprouvons, il s'ensuit que nous n'avons pas la liberté de ne pas être normatifs, de ne pas subsumer de nouvelles situations à ce que nous anticipons. Mais, nous sommes libres de réfléchir sur nos anticipations spontanées, et, de manière générale, sur les structures qui pré-organisent notre expérience. Cette liberté n'est pas le contraire de la normativité, elle en est l'envers.

${ }^{1}$ Ibid., p. 520 : la capacité d'assumer «ce que je suis par hasard» et d'«aller audelà ». 